HEFAT2012

$9^{\text {th }}$ International Conference on Heat Transfer, Fluid Mechanics and Thermodynamics

16 - 18 July 2012

Malta

\title{
TWO-PHASE FLOW CHARACTERISTICS IN SINGULARITIES
}

\author{
Revellin R.* and M. Padilla and Bonjour J. \\ *Author for correspondence \\ CETHIL UMR 5008 \\ INSA Lyon \\ Bât Sadi Carnot \\ 9 Rue de la Physique \\ 69621 Villeurbanne cedex \\ France \\ E-mail: remi.revellin@insa-lyon.fr
}

\section{ABSTRACT}

This paper aims at presenting the latest scientific progress on two-phase flow in singularities through academic research at INSA Lyon as well as proposing some future possible important issues to be investigated. Flow regimes of third- and fourth-generation refrigerants in horizontal and vertical return bends as well as in a horizontal sudden contraction were experimentally investigated. The dynamical behavior of vapor bubbles or slugs in vertical downward flow return bend was reported. A simplified analysis of the forces acting on the bubble was proposed to better understand the vapour trajectory. Furthermore, void fraction was measured along the sudden contraction using an image analysis technique, which gives very original results.

Such experimental studies also brought to the fore the upstream and downstream flow disturbances caused by such singularities as contractions and return bends and their impact on the hydrodynamic performance (e.g. pressure drop) of refrigerants. Especially, these disturbances can be analysed in terms of perturbation lengths up- and downstream of the singularities.

Lastly, large pressure drop databases for R-410A, R-134a and HFO-1234yf were obtained. Experimental values of pressure drops in singularities were compared against different prediction methods from the literature without any satisfactory results. Finally, these databases were used to develop new twophase pressure drop prediction methods for such singularities as return bends and sudden contractions.

\section{INTRODUCTION}

Singularities, such as return bends and sudden contractions, are extensively used in compact refrigeration systems such as air conditioners or heat pumps. Either single-phase or twophase flow can occur in these applications. Two-phase pressure drops in return bends and in sudden contractions in refrigeration systems have been experimentally investigated by several authors in the open literature. Since the 60's, several refrigerants have been studied, among which CFC, HCFC and HFC as well as air and water. Most recent researches on return bends have been carried out for R-134a and R-410A whereas for sudden contractions the working fluids were air and water. Nevertheless, there is a growing interest in refrigeration, heat pump and air conditioning industries to protect the environment from refrigerants with high Global Warming Potential (GWP). This has led to a demand of new environmentally friendly refrigerants. In this sense, the HydroFluoroOlefin (HFO) 1234 yf, with a GWP of 4 , is presented as a solution able to provide efficient and effective cooling with a near drop-in replacement for the current refrigerant R-134a. Among its interesting characteristics, HFO-1234yf has an atmospheric lifetime of only 11 days, compared to 13 years for R-134a.

In this paper, we present the latest scientific progress carried out on two-phase flow in singularities through academic research at INSA Lyon as well as proposing some future possible important issues to be investigated. The results presented in this work are for the following fluids: R-134a, R410A and HFO-1234yf.

\section{NOMENCLATURE}

$\begin{array}{lll}a, b & {[\mathrm{~m}]} & \text { Vapor cross section lengths } \\ D & {[\mathrm{~m}]} & \text { Inner diameter } \\ G & {\left[\mathrm{~kg} / \mathrm{m}^{2} \mathrm{~s}\right]} & \text { Mass velocity } \\ K & {[-]} & \text { Correlation parameter } \\ P & {[\mathrm{~Pa}]} & \text { Pressure } \\ R, r & {[\mathrm{~m}]} & \text { Curvature radius } \\ T & {\left[{ }^{\circ} \mathrm{C}\right]} & \text { Temperature } \\ x & {[-]} & \text { Vapour quality } \\ & & \\ \text { Special characters } & \\ \varepsilon & {[-]} & \text { Void fraction } \\ \theta & {[\mathrm{deg}]} & \text { Return bend curvature angle } \\ \rho & {\left[\mathrm{kg} / \mathrm{m}^{3}\right]} & \text { Density }\end{array}$


Subscripts

$\begin{array}{ll}\text { c } & \text { Contraction } \\ \text { curv } & \text { Curvature } \\ \text { in } & \text { Inlet } \\ 1 & \text { Liquid } \\ \mathrm{m} & \text { Momentum } \\ \text { out } & \text { Outlet } \\ \text { pert } & \text { Perturbation } \\ \text { rb } & \text { Return bend } \\ \text { sat } & \text { Saturation } \\ \text { sing } & \text { Singular } \\ \text { st } & \text { Straight tube } \\ \text { tot } & \text { Total } \\ \text { v } & \text { Vapor }\end{array}$

\section{TEST FACILITY}

Erreur ! Source du renvoi introuvable. shows a sketch of the refrigerant circuit. It consists of two parallel loops: the refrigerant loop and the water-glycol loop. The test facility is designed to make it possible single-phase and twophase tests using various refrigerants. The refrigerant flow loop consists of a gear pump which delivers sub-cooled refrigerant to the heater. The refrigerant is preheated and partially evaporated in the heater to the desired vapor quality. The vapor quality entering the test section is calculated from the energy balance on the electrical heater. Then the fluid flows through the condenser, the liquid reservoir and the subcooler before returning to the pump. An energy balance has been performed to ensure accurate measurements of the vapour quality. In addition, single-phase pressure drop tests have been carried out to check the reliability of the two-phase pressure drop measurements.

The complete description of the test facility can be found in [1] and [2].

\section{DATA REDUCTION}

In order to obtain the values of the vertical or horizontal return bend pressure drop $\Delta P_{\mathrm{rb}}$, the total pressure drop $\Delta P_{\text {tot }}$ and the horizontal straight tube pressure drops $\left(\Delta P_{\text {st,in }}\right.$ and $\left.\Delta P_{\text {st,out }}\right)$, have been measured. Figure 2 shows a synthesis of the idealized pressure profile of the flow path along a vertical return bend (most general case). $\Delta P_{\text {tot }}$ corresponds to the total pressure drop experimentally measured over the length $L_{i o}$. The regular pressure drop $\left(\Delta P_{\text {st,in }}\right.$ and $\left.\Delta P_{\text {st,out }}\right)$ has been removed from the total pressure drop $\Delta P_{\text {tot }}$ to obtain the return bend pressure drop $\Delta P_{\mathrm{rb}}$ :

$$
\Delta P_{\mathrm{rb}}=\Delta P_{\text {tot }}-\Delta P_{\mathrm{st}, \text { in }}-\Delta P_{\mathrm{st}, \mathrm{out}}
$$

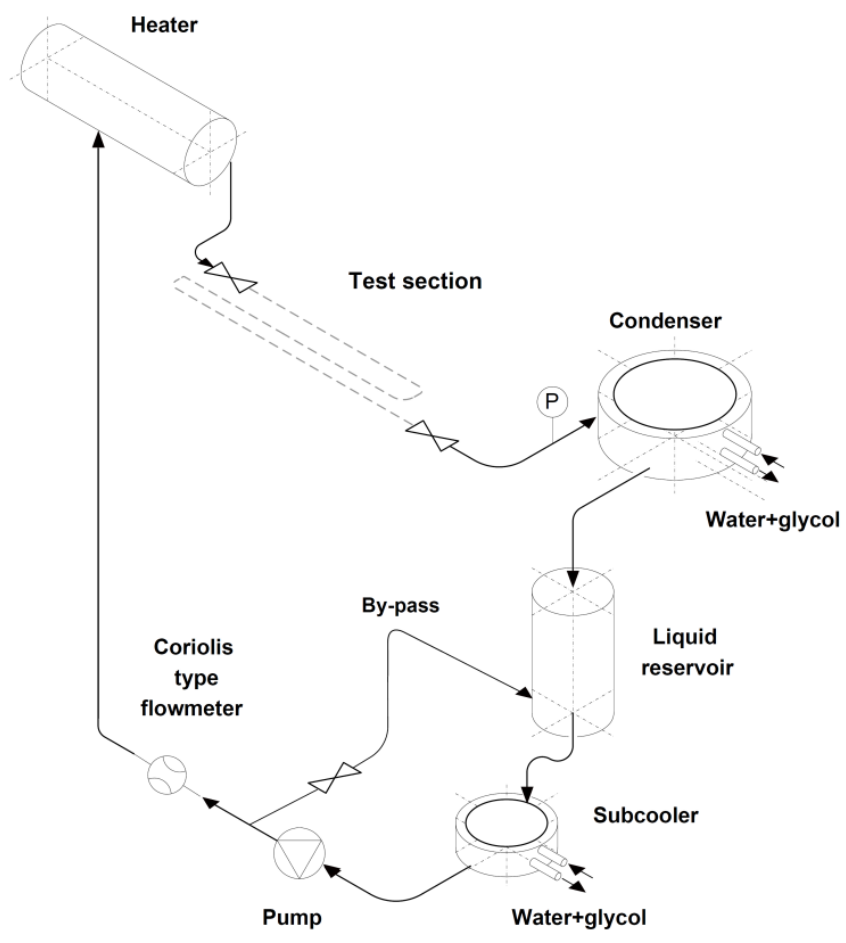

Figure 1: Test facility

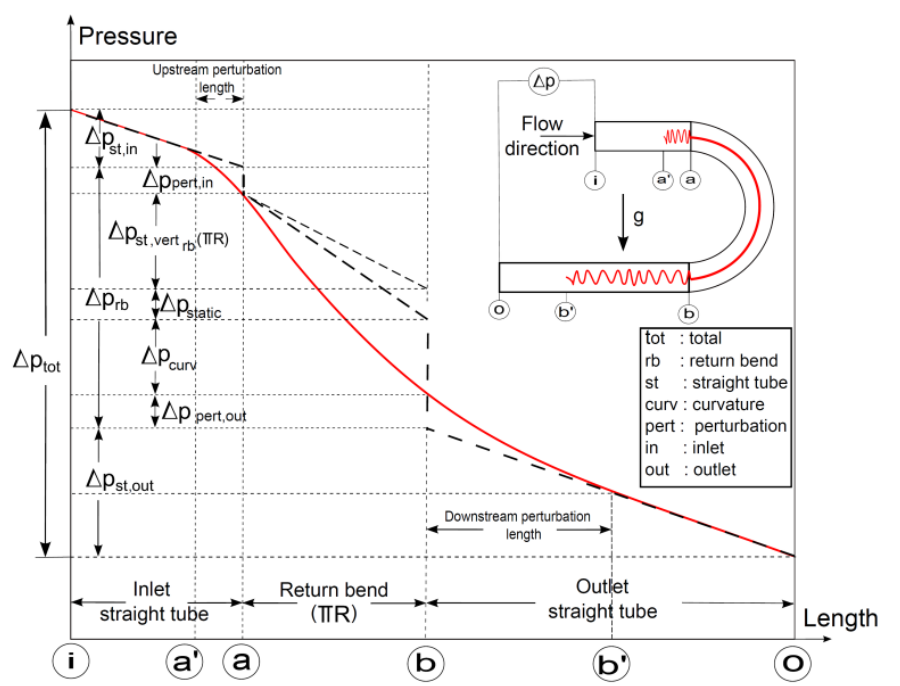

Figure 2: Idealized pressure profile along a return bend

In order to obtain the values of the sudden contraction pressure drop $\Delta P_{\mathrm{c}}$, the total pressure drop $\Delta P_{\text {tot }}$ and the straight tube pressure drops $\left(\Delta P_{\text {st,in }}\right.$ and $\left.\Delta P_{\text {st,out }}\right)$ have been measured. Figure 3 shows a synthesis of the idealized pressure profile along the flow path in a sudden contraction. The regular pressure drop up- and downstream of the sudden contraction has been removed from the total pressure drop to obtain the sudden contraction pressure drop $\Delta P_{\mathrm{c}}$.

$$
\Delta P_{\mathrm{c}}=\Delta P_{\text {tot }}-\Delta P_{\text {st,in }}-\Delta P_{\text {st,out }}
$$




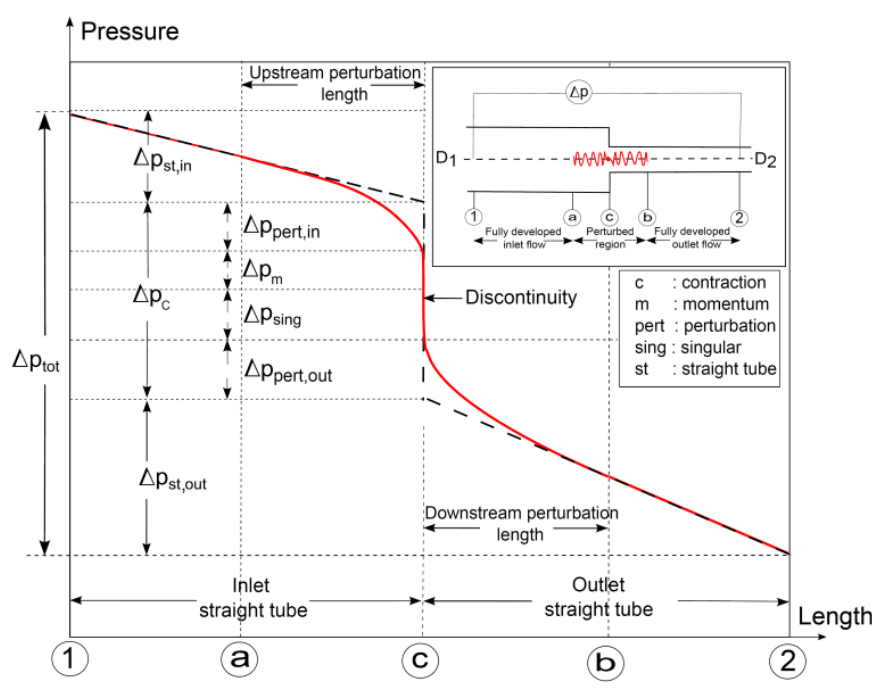

Figure 3: Idealized pressure profile along a sudden contraction

\section{VISUAL OBSERVATIONS, BUBBLE DYNAMICS AND VOID FRACTION}

\section{Return bend}

The different flow patterns were found to be characterized by distinct dynamical bubble behaviors. In general, when a bubble or a vapor slug travels along the curved section of the return bend, it first slides on the outer side tube wall for a distance, and then detaches. After a period of movement inside the curved section, it re-attaches to the inner side tube wall and then slides on the upper part of the tube wall along the liquid flow direction.

In slug flow regime, the observed bubbles have an equivalent radius lower than $4 \mathrm{~mm}$. The bubbles slide for a very short distance (see Figure 4(a)) and then they depart away from the outer side tube wall before an angular position of about $45^{\circ}$. Similar observations have been carried out by [3] with R-141b. The small bubbles (equivalent radius $<1.5 \mathrm{~mm}$ ) are observed to follow an arc route. These bubbles detach from the outer side tube wall before a angular position $70^{\circ}$. For the present study, the detachment point is not superior to $50^{\circ}$.

Figure 4(a) presents an image reconstitution (time lapse between images $15 \mathrm{~ms}$ ) which allows to visualize the trajectory of a small bubble of HFO-1234yf with an equivalent radius around of $0.5 \mathrm{~mm}$ moving along the return bend. As can be observed the bubble slides from the outlet of the horizontal tube to the outer side wall of the curved tube. The bubble detaches at approximately $30^{\circ}$ and then takes off. After a period of movement inside the curved section (between $40^{\circ}$ and $130^{\circ}$ ), it re-attaches to the inner side tube wall at about $130^{\circ}$ and slides on the tube wall along the liquid flow direction.

Concerning the vapor slug behavior, although the size of the vapor phase is bigger, the detachment still happens before $50^{\circ}$. When the equivalent bubble radius is greater than $3 \mathrm{~mm}$, the vapor slug is relatively long and the detachment process is separated into two different parts, one concerning the vapor slug head detachment and the other regarding the vapor slug tail detachment.
The information available in the literature related to slug flow is only for straight tubes under various orientations, not Ubends. In inclined straight tubes, very little attention has been given to downward gas-liquid flows. [4] carried out a study on the effect of pipe inclination angle $(\theta)$ focused on the slug flow regimes. The pipe inclination angles considered were from $7.5^{\circ}$ to $-1^{\circ}$. Results show that the pipe inclination affects mainly the bubble length, while the liquid slug length remains insensitive to the variation of the inclination angle. The slug head is affected by the pipe's inclination and gas flow rate, while the shape of the slug tail seems to remain approximately normal to the pipe axis for all experimental conditions considered.

In order to visualize the vapor trajectory along the return bend, a superposition of 7 images (time lapse between images $60 \mathrm{~ms}$ ) for vapor slug head (Figure 4(b)) and vapor slug tail (Figure 4(c)) of HFO-1234yf have been constructed. Both head and tail depart from the outer side tube wall at different positions. Normally, vapor slug head is lifted up at about $80^{\circ}$ of the bend, leaving a liquid film between it and the outer side tube wall. Sometimes the vapor slug head re-attaches to the inner side tube wall even before the vapor slug tail detaches the outer side tube wall.

An analysis of the forces acting on the bubble was performed by [5] to better understand these observations. The forces are applied at the gravity center of a moving small vapor bubble induced by the flow field with a non uniform pressure gradient (Figure 5), where the bubble experiences (i) buoyancy due to gravity (which comprises the effect of the bubble weight in the opposite direction); (ii) hydrodynamic drag which is opposed to the relative motion of the bubble through the liquid phase; (iii) a hydrodynamic lift force due to the shear within the liquid phase which is perpendicular to the flow direction; and (iv) buoyancy caused by the reaction of the liquid phase on the bubble due to the effect of the centrifugal acceleration acting on the liquid phase (which also comprises the effect of the centrifugal acceleration acting on the vapor phase in the opposite direction).

The latter, is also known as "apparent buoyancy" and is described by [6]. When the bubble touches the tube wall, a new force due to the contact pressure appears in the forces balance at the center of the bubble. A convenient strategy to identify this force is presented by [7] by considering a bubble footed at a plane wall and symmetrical about the axis perpendicular to the wall through the center of the bubble. The time rate of change of the kinetic energy in the liquid is computed with the mechanical energy balance. This force represents the hydrodynamic force component of the bubble normal to the wall, and it depends on the dynamic contact angle and surface tension.

This analysis allows to better understand the effect of the various forces acting on the vapor phase. The detachment is mainly controlled by the apparent buoyancy and hydrodynamic lift. The re-attachment is controlled by the apparent buoyancy and the radial component of buoyancy due to gravity. In general, the secondary flow in the liquid phase influences the trajectory of the small bubbles while it is probably negligible in the case of vapor slugs. As mentioned before, after detaching 
from the outer side tube wall, bubbles and vapor slugs experience a period of movement inside the tube before they reattach to the inner side tube wall. Note that the re-attachment of the slug head happens even before the detachment of the slug tail when the vapor slug is long enough.

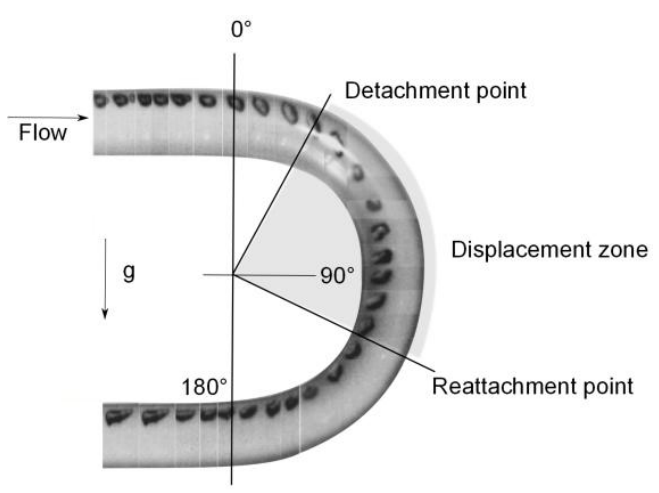

(a) Bubbly flow

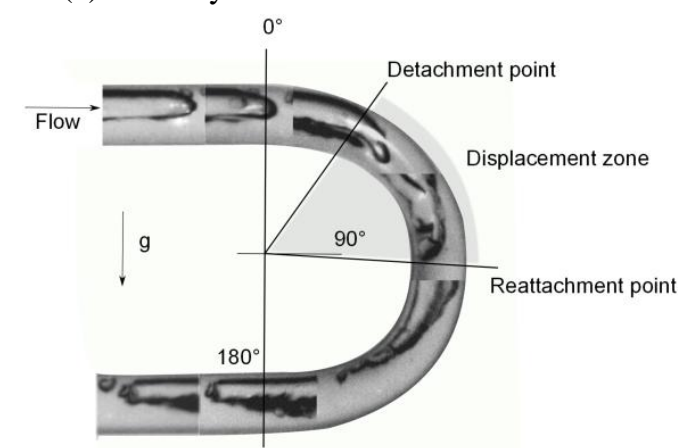

(b) Slug head

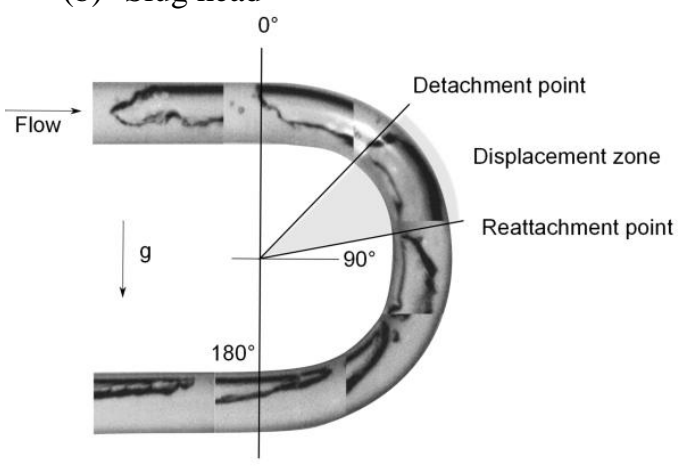

(c) Slug tail

Figure 4: Visual reconstitution of bubble, slug head and slug tail behaviors and trajectories during a vertical downward flow

in a return bend. Test conditions are $x=5 \%, G=300 \mathrm{~kg} / \mathrm{m}^{2} \mathrm{~s}$, $T_{\text {sat }}=10^{\circ} \mathrm{C}$ and $D=6.7 \mathrm{~mm}$.

Bubble detachment positions as a function of the bubble equivalent radius for HFO-1234yf and R-134a are plotted in Figure 5a in an analogous manner as in [5]. Since the shape of a bubble is rather complex during its travel along the return bend, the bubble size is calculated before the bend entrance by an image processing program which gives the bubble projected area. This procedure is carried out along three different locations upstream of the return bend. As a result, the bubble equivalent radius considered in this study corresponds to the radius of an equivalent disk with exactly the same projected area.

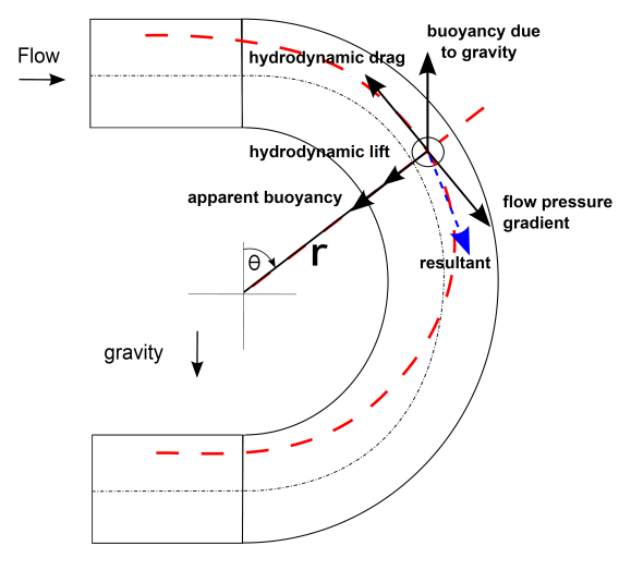

(a) detachment

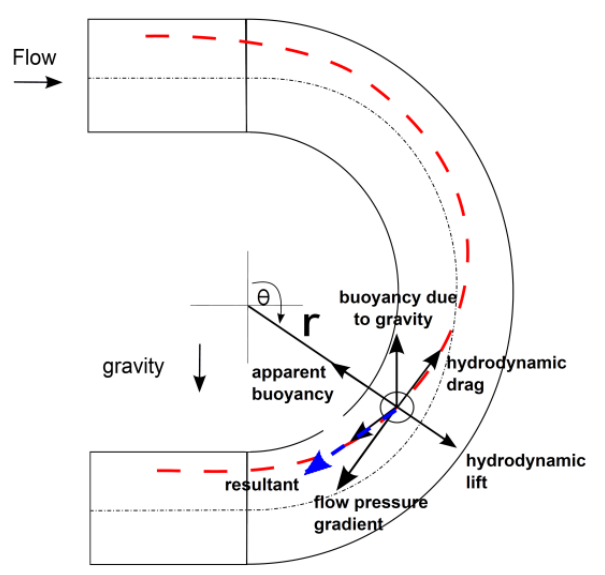

(b) re-attachment

Figure 5: Forces acting on a moving vapor bubble along a return bend

This figure also presents the results obtained by [3] for R-141b as comparison. The general trend is that bigger bubbles detach later (at greater angle) than smaller ones. Considering a very small bubble, it is reasonable to believe that secondary flow would be strong enough to detach it from concave part of the curved tube before $40^{\circ}$, and further dominate its trajectory after departure.

It is also noted that for the same bubble size, the detachment point for R-134a occurs before that for HFO-1234yf, and both before those observed for R-141b. Although the temperature range and data reduction for $\mathrm{R}-141 \mathrm{~b}$ are different from that for HFO-1234yf and R-134a, the effect of the fluid on the detachment behavior is clearly shown. Figure 6(b) presents the bubble re-attachment positions as a function of the bubble equivalent radius for HFO-1234yf and R-134a. Results suggest that the re-attachment of the biggest bubbles occurs at a lower angle than for smaller ones. 


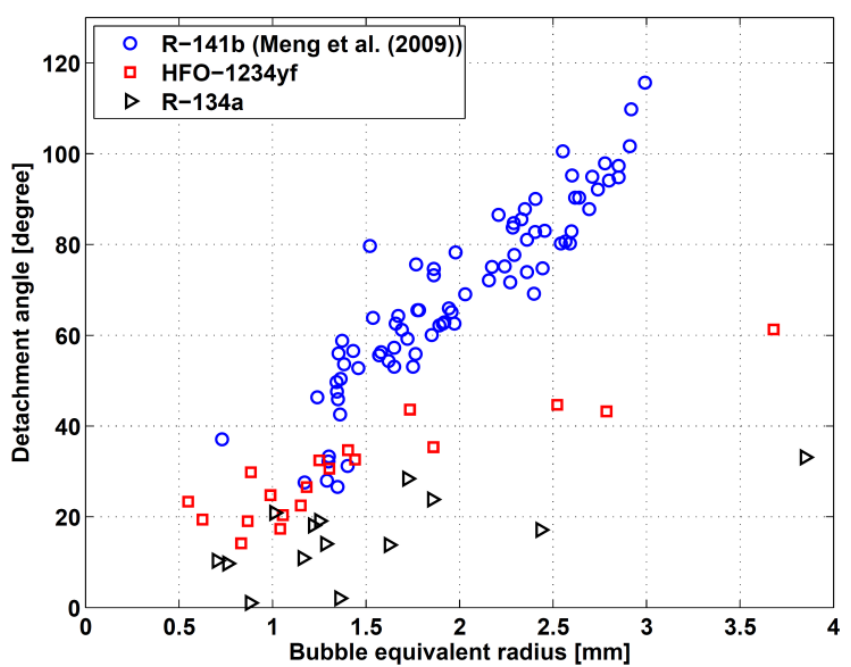

(a) Bubble detachment position vs. bubble equivalent radius

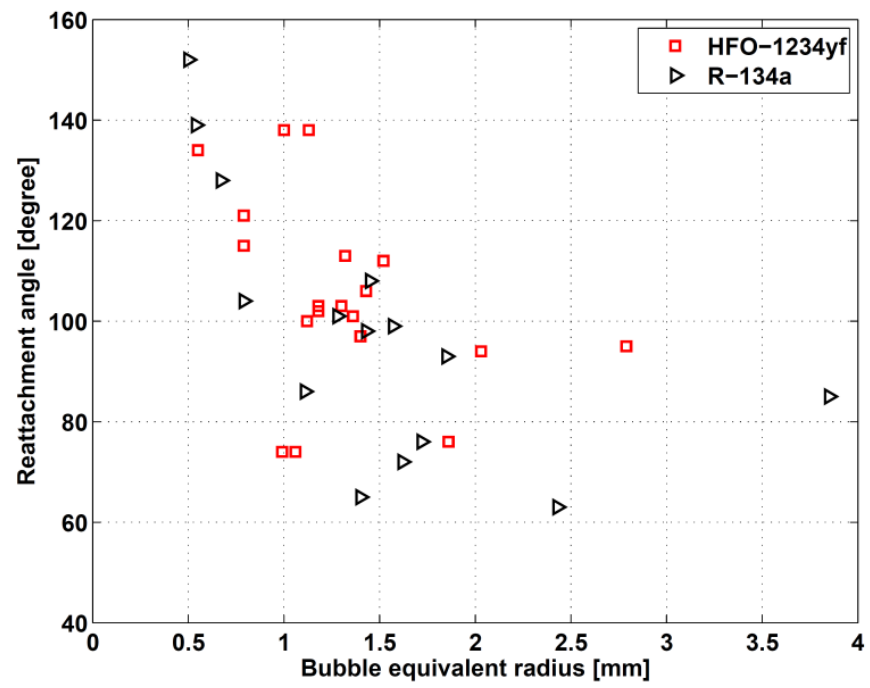

(b) Bubble re-attachment position vs. bubble equivalent radius

Figure 6: Location of bubble detachment and re-attachment vs. bubble equivalent radius. Test conditions are $x=5 \%, G=300$ $\mathrm{kg} / \mathrm{m}^{2} \mathrm{~s}, T_{\text {sat }}=10^{\circ} \mathrm{C}$ and $D=6.7 \mathrm{~mm}$.

\section{Sudden contraction}

A mirror, placed at $45^{\circ}$, was used to visualize simultaneously the top and the side views of the sudden contraction [8]. Figure 7(a) presents the evolution of a small bubble of $\mathrm{R}-134 \mathrm{a}$ (approx. equivalent radius $1.0 \mathrm{~mm}$ ) moving along the sudden contraction. As can be observed, the side view reveals the effect of buoyancy on the bubble which slides from the horizontal inlet tube attached to the top side wall of the tube. This behavior continues beyond the sudden contraction in the outlet tube following the liquid flow direction. The top view shows the bubble trajectory almost centered in the tube. The effect of the acceleration due to a change of cross-section in the singularity can be observed as the bubble elongates when passing through the singularity.

Figure 7(b) and Figure 7(c), present the evolution of a vapor slug head and vapor slug tail respectively. As can be noted in Figure 7(b), the vapor slug head elongates when passing along the singularity due to the effect of the flow acceleration. It is also observed that the vapor slug head recovers its rounded shape after a distance of $3 \mathrm{D}_{2}$ of the singularity. Regarding the vapor slug tail behavior, Figure 7(c) shows that when the thickness of the tail is small, the tail detaches from the vapor slug, leaving small bubbles behind it.

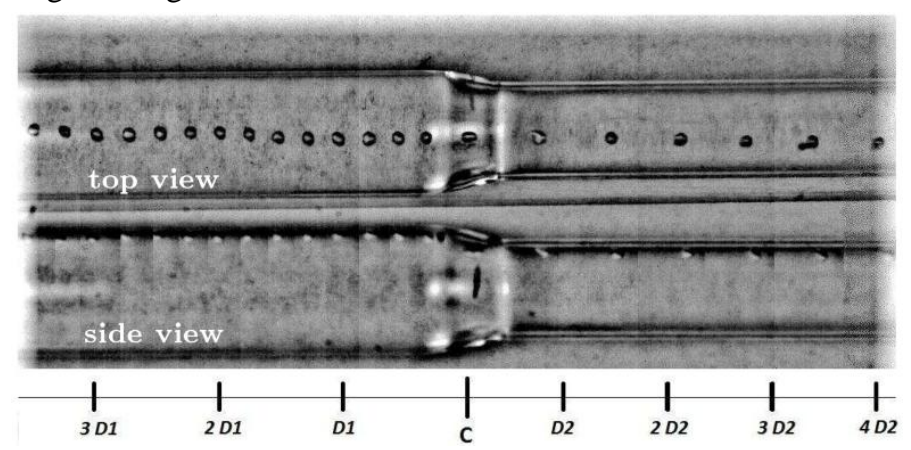

(a)

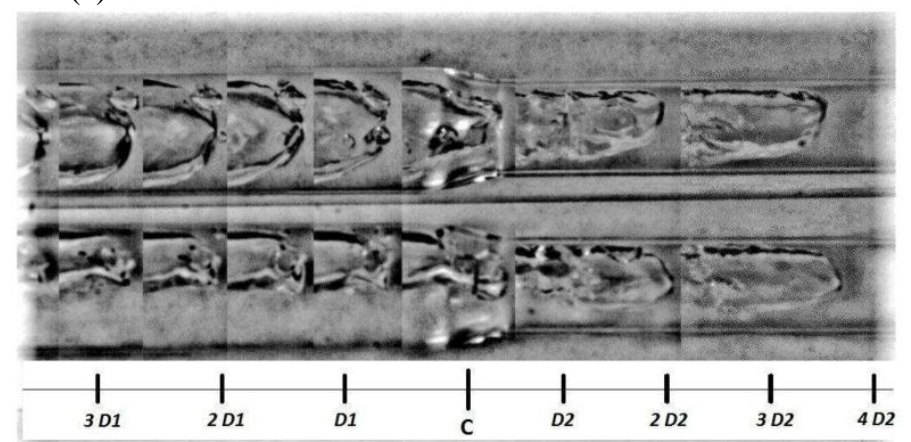

(b)

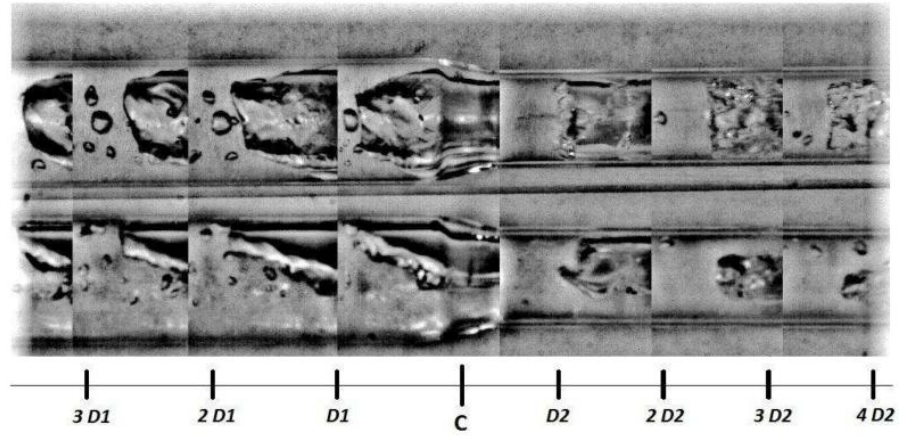

(c)

Figure 7: Visual reconstitution of bubble, slug head and slug tail behaviors and trajectories along a sudden contraction for $x=2 \%, G_{1}=500 \mathrm{~kg} / \mathrm{m}^{2} \mathrm{~s}, T_{\mathrm{sat}}=9^{\circ} \mathrm{C}, D_{1}=10 \mathrm{~mm}$ and $\sigma_{A}=0.49$.

The simultaneous top and side visualizations allow to measure the space occupied by the vapor phase over a tube crosssection. Assuming the cross section of the vapour flow to be elliptic, the void fraction can be estimated (Figure 8). This measurement has never been done in the literature so far. 

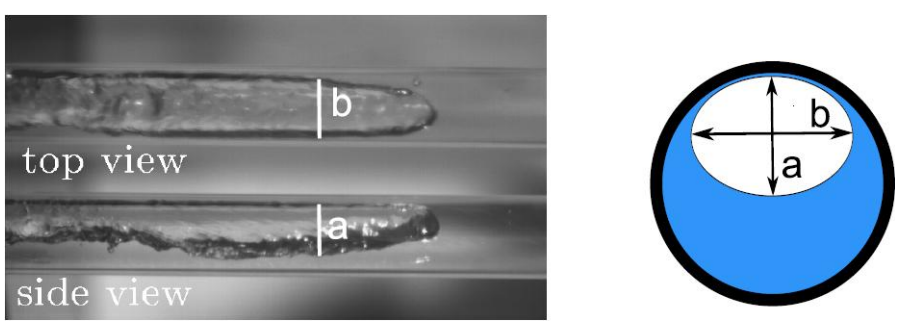

Figure 8 : (a) Top and side view of a refrigerant two-phase flow; (b) Approximation of the cross section

Figure 9 shows that the void fraction decreases when approaching the sudden contraction and increases upstream. The model by [21] Rouhani S. Z. and Axelsson E., Calculation of volume void fraction in subcooled and quality region, International Journal of Heat and Mass Transfer, Vol. 13, 1970, pp. 383-393.seems to be more accurate at predicting the void fraction than the homogeneous model. These tendencies must be confirmed by new tests but it seems that the assumption about the homogeneous flow made by different authors in the literature should be revised. These first results are very encouraging and the effort in understanding the void fraction change and bubble dynamics along a sudden contraction should be pursued. The same method could be applied to a return bend since the measurement of void fraction along the curvature has never been investigated.

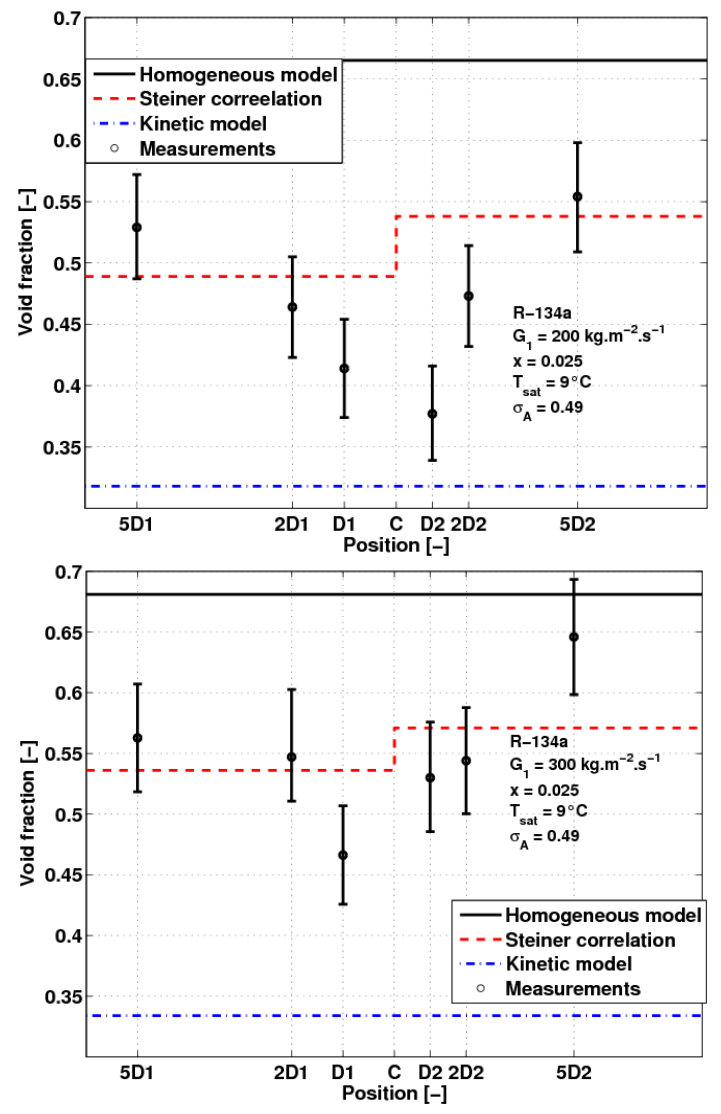

Figure 9: Void fraction measurements along a sudden contraction.

\section{PERTURBATION LENGTHS}

The perturbation lengths are an important issue in singularities to understand the mechanisms involved in the pressure drop.

To determine the perturbation lengths up- and downstream of the vertical and horizontal return bends (sections a-a' and bb', see Figure 2, some experimental tests have been performed.

A comparison between the return bend pressure drop measured at different pressure tap locations up- and downstream for vertical and horizontal return bends is presented in Figure 10. Concerning the perturbation length upstream of the return bend, in both cases (horizontal and vertical) it is noted a significant difference in the return bend pressure drop between the pressure taps located at $5 D$ and $10 D$. In both horizontal and vertical cases, it is noted that there is no significant difference in the return bend pressure drop between any positions from $20 D$ to $50 D$ downstream of the return bend. The positions affected by the perturbations are represented by a filled marker.

In the literature, different values of the perturbation lengths up- and downstream of the return bend have been reported. Generally, the results are of the order of magnitude of our values $[9,[10]$ but sometimes, the measurements are much different. This is the case of [11] who found a downstream perturbation length up to $100 \mathrm{D}$. The most difficult in this investigation is to quantify the difference between two measurements ([2[5] used the Wilcoxon matched-pairs signed rank tests for two-related samples) and to measure the entire pressure drop including all the perturbation.

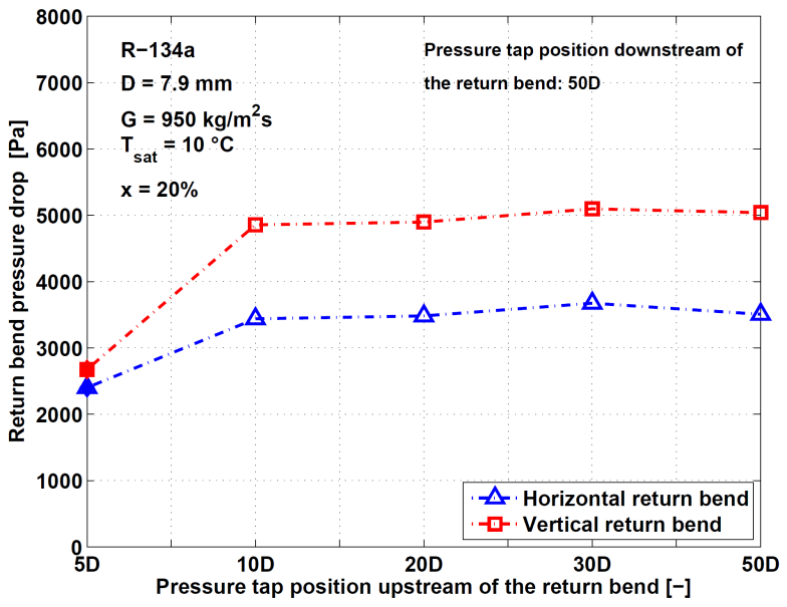

(a) Upstream 


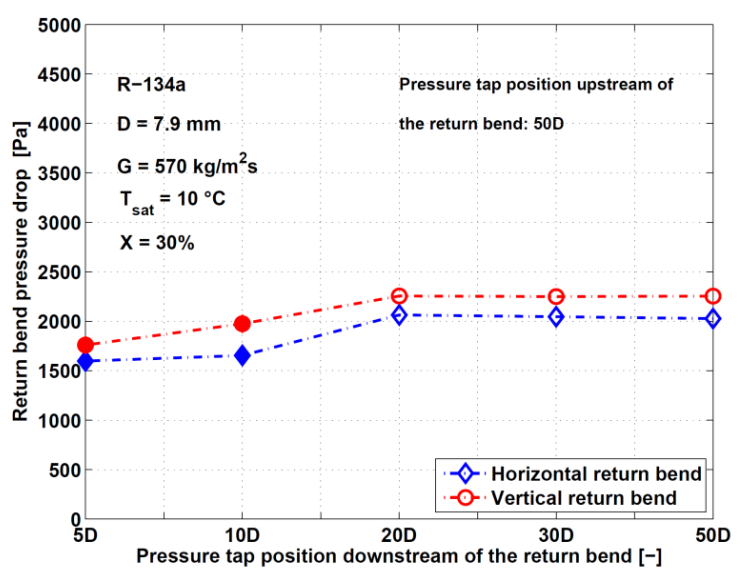

(b) Downstream

Figure 10: Return bend pressure drop measured at five different pressure tap positions upstream and downstream of the horizontal and vertical return bend. Filled markers correspond to measurements affected by the perturbation.

Figure 11 shows the sudden contraction pressure drop $\left(\Delta \boldsymbol{P}_{\mathbf{c}}\right)$ for R-410A for various locations of the upstream and downstream pressure tap [12]. For these conditions, there was no significant difference in the sudden contraction pressure drop between each pressure tap position above $28 \mathrm{D}_{2}$ upstream of the singularity and $70 \mathrm{D}_{2}$ downstream. The positions affected by the perturbations are represented by a filled marker.

In the future, the effect of the curvature radius on the perturbation length should be investigated for the return bend. For the sudden contraction, where there is a lack of experimental data for refrigerants, the effect of the tube orientation and the $\sigma_{A}=D_{2} / D_{1}$ ratio should be investigated.

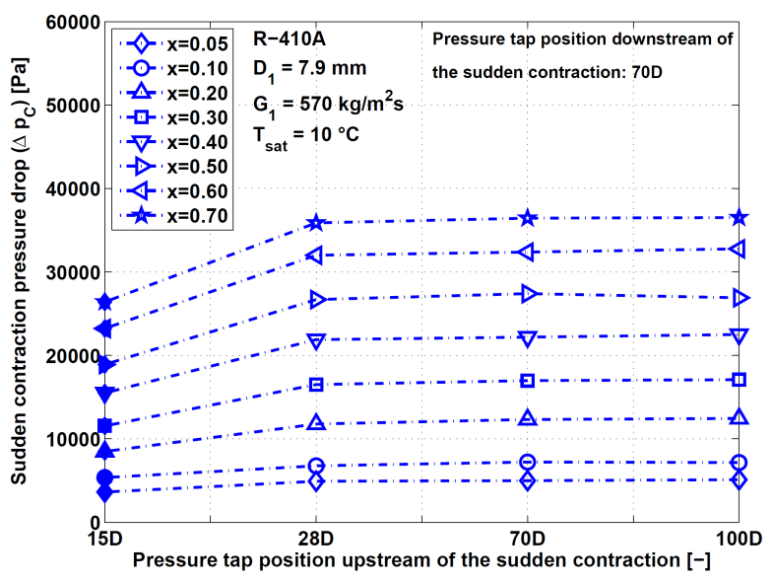

(a) Upstream

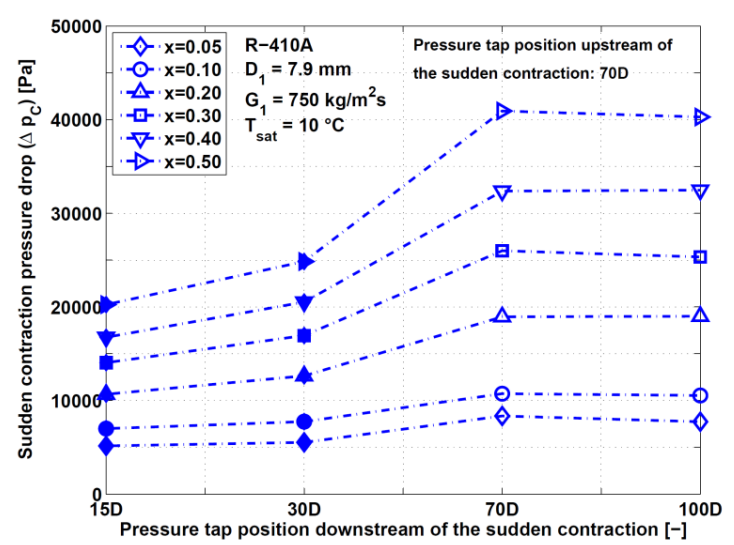

(b) Downstream

Figure 11: Sudden contraction pressure drop measured at four different pressure tap positions upstream and downstream of the horizontal sudden contraction. Filled markers correspond to measurements affected by the perturbation.

\section{PRESSURE DROP RESULTS}

Figure 12: Return bend pressure drop: effect of refrigerant and tube orientation. presents a comparison between refrigerants HFO-1234yf, R-410A and R-134a. Results show that the pressure drop for HFO-1234yf is lower than that for R-134a. This behavior is the consequence of the physical properties of the refrigerants and is independent of the return bend orientation. This figure also shows the orientation effect for the three refrigerants at the same experimental conditions. In general, vertical return bend pressure drops are higher than that observed for horizontal orientation. The maximum pressure drop occurs around $60 \%$ of vapor quality regardless of orientation. According to the flow pattern map of [13], this maximum occurs for annular flow regime at the inlet of the bend.

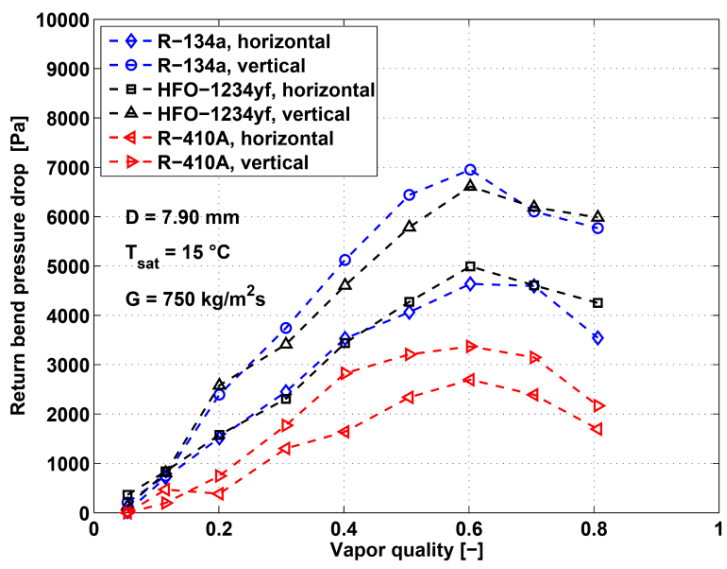

Figure 12: Return bend pressure drop: effect of refrigerant and tube orientation.

The 1087 pressure drop data points measured in horizontal and vertical return bends $\left(\Delta \boldsymbol{P}_{\mathbf{r b}},\right)$ for refrigerants HFO-1234yf, R$134 \mathrm{a}$ and R-410A were compared against four two-phase 
frictional pressure drop prediction methods in return bends [14 [15[16[17[18]. In general, none of the prediction methods allow to explain the phenomena involved during two-phase flow in return bends. Based on the observations made in this study, the intention was to develop a new method for predicting the pressure drop in return bends using a different approach from those used in other studies. The new method is called mainly to predict the return bend pressure drop $\left(\Delta \boldsymbol{P}_{\mathbf{r b}},\right)$ from the contributions of all the effects previously discussed (e.g friction, curvature, orientation, perturbation lengths, etc.). This new method [19] has been compared against the present database and the data points from the literature.

$\Delta \boldsymbol{P}_{\text {rb }}=\Delta \boldsymbol{P}_{\text {st }}+\Delta \boldsymbol{P}_{\text {static }}+\Delta \boldsymbol{P}_{\text {sing }}$

where

$\Delta P_{\text {st }}$ is calculated by the relation proposed by [20]. $\Delta P_{\text {static }}$ is calculated with the conventional relation using the void fraction model [21]. $\Delta P_{\text {sing }}$ is given by the following relation:

$\Delta P_{\text {sing }}=K_{\mathrm{l}} \Delta P_{1}+K_{\mathrm{v}} \Delta P_{\mathrm{v}}$

where

$K_{\mathrm{l}}=3\left(\mathrm{x}^{3}+(1-\mathrm{x})+0.055(1-\mathrm{x})^{3}\right)\left(\frac{\mathrm{D}}{2 \mathrm{R}}\right)^{1 / 3}$
$K_{\mathrm{v}}=3\left(\mathrm{x}^{3}(0.055-(1-\mathrm{x}))\right)\left(\frac{\mathrm{D}}{2 \mathrm{R}}\right)^{1 / 3}$
$\Delta P_{\mathrm{l}}=\frac{\mathrm{G}^{2}(1-\mathrm{x})^{2}}{\rho_{\mathrm{l}}(1-\varepsilon)^{2}}$
$\Delta P_{\mathrm{v}}=\frac{\mathrm{G}^{2} \mathrm{x}^{2}}{\rho_{\mathrm{l}} \varepsilon^{2}}$

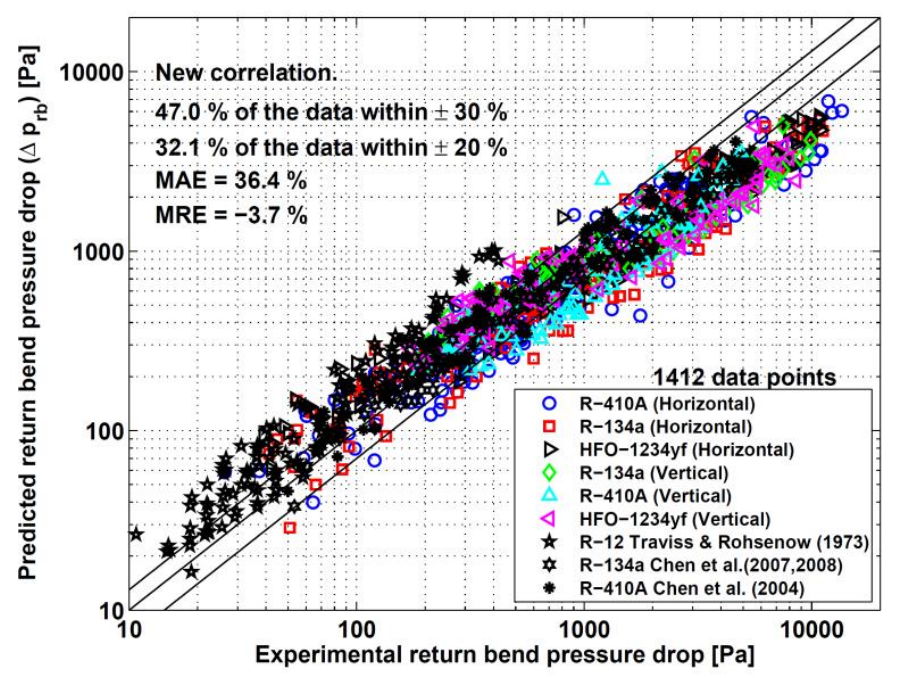

Figure 13: New correlation for return bend pressure drop compared against the present experimental data base and that from the literature.

This new method presents many advantages:

- It contains only two empirical constants.

- It has been developed based on a large database (1412 data points) including the fourth generation HFO-1234yf and the well know third generation R-134a, R-410A and R-12 refrigerants in both horizontal and vertical orientations.

- Based on the visual observations, various effects including centrifugal forces acting on both phases and buoyancy due to gravity have been considered in the formulation of the new method.

- This correlation does not assume a homogeneous flow.

-The physical limits in the new method are correct. As a matter of fact, when $R \rightarrow D / 2$, the effect of the curvature on the singular pressure drop reaches its maximum value, and when $R \rightarrow \infty, D / 2 R \rightarrow 0$, giving $\Delta P_{\text {sing }}=0$. In addition, when $\mathrm{x}=0$ or $\mathrm{x}=1$, the resulting pressure drop tends toward the single phase pressure drop in return bend.

- The relation has been developed considering commercial tube diameters from 7.9 to $10.85 \mathrm{~mm}, 2 R / D$ from 3.68 to 4.05 , a wide range of mass velocities (189 to $1695 \mathrm{~kg} / \mathrm{m}^{2} \mathrm{~s}$ ) and saturation temperatures from $4.4^{\circ} \mathrm{C}$ to $22^{\circ} \mathrm{C}$, which constitute a relatively large range of experimental conditions.

- The term $\Delta P_{\text {static }}$ represents the contribution of the static pressure drop given by a vertical orientation.

For the sudden contraction, the comparison between the existing prediction methods [[22[14[23[24[25] and the present database (360 data points) led to poor results. A new correlation has been proposed by [12] and is written as follows:

$$
\Delta P_{\mathrm{c}}=\Delta P_{\text {pert }}+\Delta P_{\mathrm{m}}+\Delta P_{\text {sing }}
$$

where $\Delta P_{\mathrm{m}}$ is calculated as follows :

$$
\Delta P_{\mathrm{m}}=\frac{\mathrm{x}^{2}}{\rho_{\mathrm{v}}}\left[\frac{\mathrm{G}_{2}^{2}}{\varepsilon_{2}}-\frac{\mathrm{G}_{1}^{2}}{\varepsilon_{1}}\right]+\frac{(1-\mathrm{x})^{2}}{\rho_{\mathrm{l}}}\left[\frac{\mathrm{G}_{2}^{2}}{1-\varepsilon_{2}}-\frac{\mathrm{G}_{1}^{2}}{1-\varepsilon_{1}}\right]
$$

The void fraction is calculated using [21].

In addition, $\Delta P_{\text {pert }}+\Delta P_{\text {sing }}$ is given by the following expression:

$$
\begin{aligned}
& K_{\mathrm{l}}=\left(2(1-\mathrm{x})\left(1-\mathrm{x}^{0.017}\right)\right)\left(1-\sigma_{A}\right)^{1 / 4} \\
& K_{\mathrm{v}}=\left(0.017 \mathrm{x}^{2}+2 \mathrm{x}^{0.017}(1-\mathrm{x})\right)\left(1-\sigma_{A}\right)^{1 / 4} \\
& \Delta P_{1}=\frac{\mathrm{G}^{2}(1-\mathrm{x})^{2}}{\rho_{\mathrm{l}}(1-\varepsilon)^{2}} \\
& \Delta P_{\mathrm{v}}=\frac{\mathrm{G}^{2} \mathrm{x}^{2}}{\rho_{\mathrm{l}} \varepsilon^{2}}
\end{aligned}
$$

The new method presents several common advantages with the previous one on return bend:

- It contains only three empirical constants.

- It has been developed based on a large database (360 data points) including the fourth generation HFO-1234yf and the well-known third generation R-134a and R-410A refrigerants.

- This correlation does not assume a homogeneous flow.

- The physical limits in the new method are correct. Note that when $\sigma_{A}=1, \Delta P_{\mathrm{c}}=0$. In addition, when $x=0$ or $x=1$, the resulting expressions for pressure drop calculation tend toward the single phase pressure drop in sudden contraction. 
- The relation has been developed considering commercial tube diameters from 5.3 to $10.85 \mathrm{~mm}, \sigma_{A}$ from 0.45 to 0.53 .

In addition, the new relation allows to calculate the different contributions:

- The term $\Delta P_{\mathrm{m}}$ represents the contribution to the pressure drop of the flow acceleration effect due to a change of cross-section.

- The term $\Delta P_{\text {pert }}+\Delta P_{\text {sing }}$ takes into account the contribution of the friction resistance due to the singularity and its perturbation effects up- and downstream on the sudden contraction pressure drop.

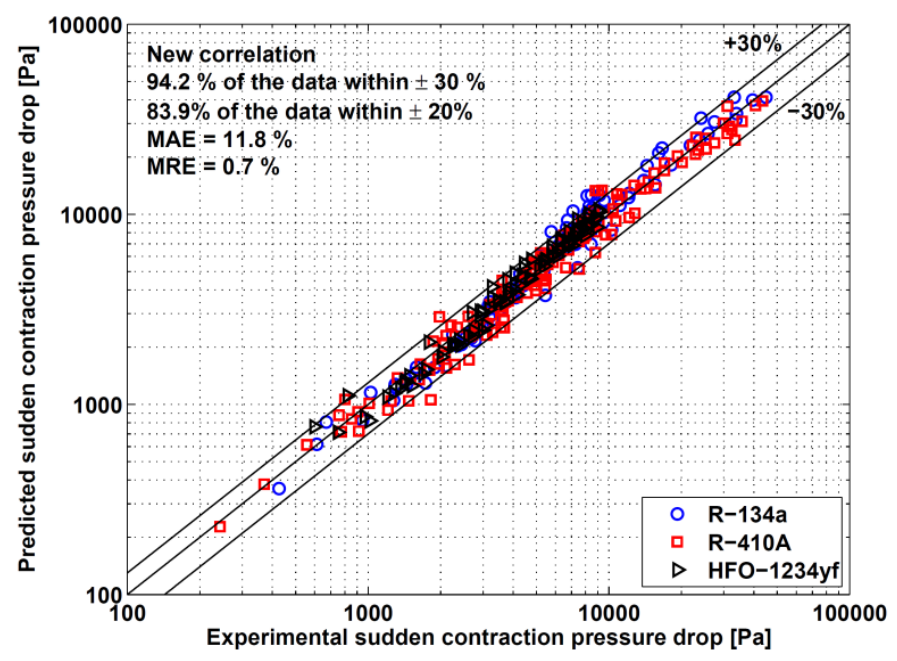

Figure 14: New correlation for sudden contraction pressure drop compared against the present experimental data base.

\section{CONCLUSION}

This paper aims at presenting the latest scientific progress on two-phase flow in singularities through academic research at INSA Lyon as well as at proposing some issues we consider as important to be investigated in the future. Flow regimes of third- and fourth-generation refrigerants in horizontal and vertical return bends as well as in a horizontal sudden contraction were experimentally investigated. The dynamical behavior of vapor bubbles or slugs in vertical downward flow return bend was reported. A simplified analysis of the forces acting on the bubble was proposed to better understand the vapour trajectory. The detachment was mainly controlled by the apparent buoyancy and hydrodynamic lift. The re-attachment was controlled by the apparent buoyancy and the radial component of buoyancy due to gravity. In general, the secondary flow in the liquid phase influences the trajectory of the small bubbles while it is probably negligible in the case of vapor slugs. Furthermore, void fraction was measured along the sudden contraction using an image analysis technique, which gives very original results. The flow deviates from the homogeneous flow contrary to what it is usually assumed in the literature for the pressure drop calculation. These first results are very encouraging and the effort in understanding the void fraction change and bubble dynamics along a sudden contraction should be pursued. The same method could be applied to a return bend since the measurement of void fraction along the curvature has never been investigated.

Such experimental studies also brought to the fore the upstream and downstream flow disturbances caused by such singularities as contractions and return bends and their impact on the hydrodynamic performance (e.g. pressure drop) of refrigerants. Especially, these disturbances can be analysed in terms of perturbation lengths up- and downstream of the singularities. For return bends, both for horizontal and vertical cases, it is noted that there is no significant difference in the return bend pressure drop above $20 D$ downstream and $10 D$ upstream of the return bend. For the sudden contraction, the results obtained showed that there is no significant difference for the sudden contraction pressure drop measured above $70 D_{2}$ downstream and above $28 D_{1}$ upstream of it. In the future, the effect of the curvature radius on the perturbation length should be investigated for the return bend. For the sudden contraction where there is a lack of experimental data for refrigerants, the effect of the tube orientation and the $\sigma_{A}=D_{2} / D_{1}$ ratio should be investigated.

Lastly, large pressure drop databases for R-410A, R-134a and HFO-1234yf were obtained. Experimental values of pressure drops in singularities were compared against different prediction methods from the literature without any satisfactory results. Finally, these databases were used to develop new twophase pressure drop prediction methods for such singularities as return bends and sudden contractions.

Void fraction, bubble dynamics, perturbation lengths and pressure drop of refrigerant two-phase flows for horizontal and non-horizontal orientation in sudden expansion and in diverging section ( $\mathrm{Y}$ - and $\mathrm{T}$ - shapes) are also important issues for the future.

However, before all this, the study of non-horizontal straight tube must be performed. As a matter of fact, straight tube pressure drop is the reference and is always removed from the total pressure drop measured along the singularity to better identify the phenomena involved.

\section{REFERENCES}

[1] Padilla M., Revellin R., Haberschill P., Bensafi A. and Bonjour J., Flow regimes and two-phase pressure gradient in horizontal straight tubes: Experimental results for HFO-1234yf, R-134a and R-410A, Experimental Thermal and Fluid Science, Vol. 35, 2011a, 1113-1126.

[2] Padilla, M., Revellin, R., Haberschill, P. and Bonjour, J., Two-phase pressure drop in return bends: Experimental results for R-410A, International Journal of Refrigeration, Vol. 34, 2011b, pp. 1854-1865.

[3] Meng M., Peng X., Ye P. and Duan Y., Bubble dynamical behavior and thermal non- equilibrium during flow boiling in U-turn bends of hairpin tubes, Chemical Engineering Process, Vol. 48, 2009, pp. 1177-1186.

[4] Roitberg E., Shemer L. and Barne, D., Hydrodynamic characteristics of gas-liquid slug flow in a downward inclined 
pipe, Chemical Engineering Science, Vol. 63, 2008, pp. 36053613.

[5] Padilla M., Revellin R. and Bonjour J., Flow regime visualization and pressure drops of HFO-1234yf, R-134a and R-410A during downward two-phase flow in vertical return bends, Submitted to International Journal of Heat and Fluid Flow in February 2012.

[6] Batchelor G., 1967, An introduction to fluid dynamics, Cambridge University Press, Cambridge.

[7] van der Geld C., The dynamics of a boiling bubble before and after detachement, International Journal of Heat and Mass Transfer, Vol. 45, 2009, pp. 831-846.

[8] Padilla M., Wallet J. Bonjour J, and Revellin R., Two-phase flow of HFO-1234yf, R-134a and R-410A in sudden contractions. Part I: Visualisation and void fraction measurements, Submitted to Experimental Thermal and Fluid Science in March 2012.

[9] Travis D.P. and Rohsenow W.M., The influence of return bends on the downstream pressure drop and condensation heat transfer in tubes, ASHRAE Semi-annual Meeting, 1973.

[10] Hoang K. and Davis M.R., Flow structure and pressure loss for two-phase flow in return bends, Transaction of ASME, Vol. 106, 1984, pp. 30-37.

[11] da Silva Lima R. J. and Thome J.R., Two-Phase Pressure Drops in Adiabatic Horizontal Circular Smooth U-Bends and Contiguous Straight Pipes (RP-1444), HVAC \& R Research, Vol. 16, 2010, pp. 383-398.

[12] Padilla M., Revellin R. and Bonjour J., Two-phase flow of HFO-1234yf, R-134a and R-410A in sudden contractions. Part II: pressure drop measurements and new prediction method, Submitted to Experimental Thermal and Fluid Science in March 2012.

[13] Wojtan L., Ursenbacher T. and Thome J.R., Investigation of flow boiling in horizontal tubes: Part I - a new diabatic twophase flow pattern map, International Journal of Heat and Mass Transfer, Vol. 48, 2005, pp.2955-2969.

[14] Chisholm D., 1983, Two-phase flow in pipelines and heat exchangers, George Godwin, London, p. 304

[15]. Idelshik IE., 1986, Handbook of Hydraulic Resistance, second ed. Hemisphere, New York, 640.

[16] Domanski P.A., Hermes C.J.L., An improved correlation for two-phase pressure drop of R-22 and R-410A in $180^{\circ}$ return bends, Applied Thermal Engineering, Vol. 28, 2008, pp. 793800 .

[17] Chen IY, Wang CC and Lin SY., Measurements and correlations of frictional single-phase and two-phase pressure drops of R-410A flow in small U-type return bends, Experimental Thermal and Fluid Science, Vol. 47, 2004, pp. 2241-2249.

[18] Padilla M., Revellin R. and Bonjour J., Prediction and simulation of two-phase pressure drop in return bends.
International Journal of Refrigeration, Vol. 32, 2009, pp. 1776-1783.

[19] Padilla M., Experimental study of third (HFC) and fourth generation (HFO) refrigerants during flow boiling in singularities, PhD Thesis, INSA de Lyon, 2011.

[20] Müller-Steinhagen H. and Heck K., A simple friction pressure drop correlation for two-phase flow pipes, Chemical Engineering Process, Vol. 20, 1986, pp. 297-308.

[21] Rouhani S. Z. and Axelsson E., Calculation of volume void fraction in subcooled and quality region, International Journal of Heat and Mass Transfer, Vol. 13, 1970, pp. 383393.

[22] Collier J. G. and Thome J. R., Convective boiling and condensation, third edition. Oxford University Press, Oxford, 1994.

[23] Abdelall E., Hahm G., Ghiaasiaan S., Abdel-Khalik S., Jeter S., Yoda M., and Sadowski D., Pressure drop caused by abrupt flow area changes in small channels, Experimental Thermal and Fluid Science, Vol. 29, 2005, pp. 425-434.

[24] Chen I. Y., Chu M.-C., Liaw J.-S., and Wang C.-C., Twophase flow characteristics across sudden contraction in small rectangular channels, Experimental Thermal and Fluid Science, Vol. 32, 2008, pp. 1609-1619.

[25] Chen I. Y., Tseng C.-Y., Lin Y.-T., and Wang C.-C., Twophase flow pressure change subject to sudden contraction in small rectangular channels, International Journal of Multiphase Flow, Vol. 35, 2009, pp. 297-306. 